\title{
Essay on Kolmogorov Law of Minus 5 over 3 Viewed with Golden Ratio
}

\author{
Ming $\mathrm{Li}^{1}$ and Wei Zhao ${ }^{2}$ \\ ${ }^{1}$ School of Information Science \& Technology, East China Normal University, No. 500, Dong-Chuan Road, Shanghai 200241, China \\ ${ }^{2}$ Department of Computer and Information Science, University of Macau, Avenue Padre Tomas Pereira, Taipa 1356, Macau
}

Correspondence should be addressed to Ming Li; ming_lihk@yahoo.com

Received 14 July 2013; Accepted 7 August 2013

Academic Editor: Carlo Cattani

Copyright (c) 2013 M. Li and W. Zhao. This is an open access article distributed under the Creative Commons Attribution License, which permits unrestricted use, distribution, and reproduction in any medium, provided the original work is properly cited.

The golden ratio is an astonishing number in high-energy physics, neutrino physics, and cosmology. The Kolmogorov $-5 / 3$ law plays a role in describing energy transfer of random data or random functions. The contributions of this essay are in twofold. One is to express the Kolmogorov $-5 / 3$ law by using the golden ratio. The other is to represent the fractal dimension of random data following the Kolmogorov $-5 / 3$ law with the golden ratio. It is our hope that this essay may be helpful to provide a new outlook of the Kolmogorov $-5 / 3$ law from the point of view of the golden ratio.

\section{Instruction}

Let $\varphi$ be the golden ratio. It equals to $(1+\sqrt{5}) / 2$. Its inverse $1 / \varphi=(\sqrt{5}-1) / 2$ is called the golden mean. Both are irrational numbers. Approximately, they are

$$
\varphi \approx 1.618, \quad \frac{1}{\varphi} \approx 0.618 \text {. }
$$

The number $\varphi$ has wide applications to various fields, ranging from physics (Wurm and Martini [1], King [2], Ding et al. [3], Feruglio and Paris [4]), to cosmology (Livio [5], Boeyens [6]).

In addition to the golden ratio, fractal is a mathematical model attracting interests of scientists and physicists in the field of high-energy physics (Ghosh et al. [7]). While studying energy transfer, the Kolmogorov $-5 / 3$ law introduced by Kolmogorov [8] plays a role in the field (Qian [9], Brun et al. [10, Chapter 7], Hillebrandt and Kupka [11, Chapter 4], Gomes-Fernandes et al. [12, page 309], Lumley and Yaglom [13], Warhaft [14], Geipel et al. [15]). Motivated by those, this essay aims at exhibiting the Kolmogorov $-5 / 3$ law and the self-similarity, which is an important fractal property (Mandelbrot [16, 17], Cattani et al. [18, 19]), from the point of the golden ratio. It is our expectation that this essay may be helpful to describe the nature of random data that follows the Kolmogorov $-5 / 3$ law.
The rest of paper is organized as follows. The preliminaries are briefed in Section 2. The results that the Kolmogorov $-5 / 3$ law and the self-similarity are explained from a view of the golden ratio are given in Section 3, which is followed by conclusions.

\section{Preliminaries}

2.1. Golden Ratio. Consider a straight line in Figure 1. Arrange three points $\mathrm{A}, \mathrm{B}$, and $\mathrm{C}$, there such that the ratio below

$$
\frac{\mathrm{AC}}{\mathrm{CB}}=\frac{\mathrm{AB}}{\mathrm{AC}}
$$

equals to $\varphi$. Then, we say that the straight line is cut in extreme and mean ratio ([5], Ackermann [20], Kaygn et al. [21]).

There are various ways to synthesize the number $\varphi$. According to the definition of extreme and mean ratio, one has

$$
x=\frac{x+1}{x} .
$$

Multiplying $x$ on the both sides of the above yields

$$
x^{2}-x-1=0 .
$$




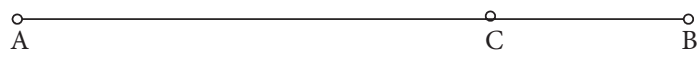

FIGURE 1: Illustration of a straight line for the golden ratio.

Solving (4) produces

$$
x_{1}=\frac{1+\sqrt{5}}{2}, \quad x_{2}=\frac{1-\sqrt{5}}{2} .
$$

Conventionally, $x_{1}$ is denoted by $\varphi$, and $x_{2}$ is denoted by $-1 / \varphi$.

2.2. Fractal Dimension. Let the autocorrelation function (ACF) of a random function $x(t)$ be $r_{x x}(\tau)$, where $r_{x x}(\tau)=$ $E[x(t) x(t+\tau)]$. Then, $r_{x x}(\tau)$ for $\tau \rightarrow 0$ represents the smallscaling phenomenon of $x(t)$. Following Davies and Hall [22], if $r_{x x}(\tau)$ is sufficiently smooth on $(0, \infty)$ and if

$$
r_{x x}(0)-r_{x x}(\tau) \sim c_{1}|\tau|^{\alpha} \quad \text { for }|\tau| \longrightarrow 0,
$$

where $c_{1}$ is a constant and $0<\alpha \leq 2$ is the fractal index of $x(t)$, the fractal dimension, which is denoted by $D$, of $x(t)$ is in the form

$$
D=2-\frac{\alpha}{2}
$$

Note that fractal dimension $1 \leq D<2$ is a measure to characterize the local self-similarity or local roughness of $x(t)$ (Mandelbrot [17], Gneiting and Schlather [23]).

Denote the power spectrum density (PSD) function of $x(t)$ by $S_{x x}(\omega)$. Denote by $\mathrm{F}$ the operator of Fourier transform. Then,

$$
S_{x x}(\omega)=\mathrm{F}\left[r_{x x}(t)\right]=\int_{-\infty}^{\infty} r_{x x}(t) e^{-j \omega t} d t, \quad j=\sqrt{-1}
$$

In the domain of generalized functions, we have (Kanwal [24], Gelfand and Vilenkin [25], Li and Lim [26])

$$
\mathrm{F}\left(|\tau|^{\alpha}\right)=-2 \sin \left(\frac{\pi \alpha}{2}\right) \Gamma(1+\alpha)|\omega|^{-(\alpha+1)},
$$

where $\Gamma$ is the Gamma function. Therefore, we have an analogy of (6) in the frequency domain in the form

$$
\mathrm{F}\left(|\tau|^{\alpha}\right) \sim c_{2}|\omega|^{-(\alpha+1)} \quad \text { for } \omega \longrightarrow \infty,
$$

where $c_{2}$ is a constant (Chan et al. [27]).

\section{Results}

The Kolmogorov -5/3 law implies that the PSD of a random function has the asymptotic behavior expressed by

$$
S_{x x}(\omega) \sim c|\omega|^{-5 / 3} \text { for } \omega \longrightarrow \infty \text {, }
$$

where $c$ is a constant (Monin and Yaglom $[28,29])$. The above well characterizes the local irregularity of turbulence function $x(t)$ (Tropea et al. [30]).
In order to connect the Kolmogorov $-5 / 3$ law with the golden ratio, we write

$$
\frac{5}{3}+e=\varphi
$$

From the previous discussions, we have

$$
e=\varphi-\frac{5}{3}=\frac{1+\sqrt{5}}{2}-\frac{5}{3}=\frac{\sqrt{5}-1}{2}-\frac{2}{3}=\frac{1}{\varphi}-\frac{2}{3} .
$$

Thus, the Kolmogorov $-5 / 3$ law expressed by (11) may be rewritten by

$$
S_{x x}(\omega) \sim c|\omega|^{-(\varphi-e)} \text { for } \omega \longrightarrow \infty
$$

From (10), we see that the fractal index may be expressed by using the golden ratio as

$$
\alpha=\varphi-e-1
$$

Thus, we attain the fractal dimension from the point of view of the golden ratio in the form

$$
D=2-\frac{\varphi-e-1}{2} .
$$

From the previous discussions, we have the following theorems.

Theorem 1. Let $x(t)$ be the random function that obeys the Kolmogorov $-5 / 3$ law expressed by (11). Then, its PSD may be expressed using the golden ratio in (14).

Theorem 2. Let $x(t)$ be the random function that obeys the Kolmogorov -5/3 law expressed by (11). Then, its fractal dimension may be represented by using the golden ratio as that in (16). The concrete value of $D$ in (16) is 5/3.

Finally, we note that the significance of the present results lies in expressing the Kolmogorov $-5 / 3$ law, which plays a role in energy transfer, by using the golden ratio, which attracts interests of researchers and scientists in high-energy physics, neutrino physics, cosmology, and many others.

\section{Conclusions}

We have explained our results in expressing the Kolmogorov $-5 / 3$ law with the golden ratio. In addition, we have expressed the fractal dimension of random data obeying the Kolmogorov $-5 / 3$ law based on the golden ratio.

\section{Acknowledgments}

This work was supported in part by the National Natural Science Foundation of China under the Project Grant nos. 61272402, 61070214, and 60873264, and by the 973 plan under the Project Grant no. 2011CB302800. 


\section{References}

[1] A. Wurm and K. M. Martini, "Breakup of inverse golden mean shearless tori in the two-frequency standard nontwist map," Physics Letters A, vol. 377, no. 8, pp. 622-627, 2013.

[2] S. F. King, “Tri-bimaximal-Cabibbo mixing," Physics Letters B, vol. 718, no. 1, pp. 136-142, 2012.

[3] G. J. Ding, L. L. Everett, and A. J. Stuart, "Golden ratio neutrino mixing and A5 flavor symmetry," Nuclear Physics B, vol. 857, no. 3, pp. 219-253, 2012.

[4] F. Feruglio and A. Paris, "The golden ratio prediction for the solar angle from a natural model with A5 flavour symmetry," Journal of High Energy Physics, vol. 2011, no. 3, article 101, 2011.

[5] M. Livio, The Golden Ration, Random House, 2003.

[6] J. C. A. Boeyens, Chemical Cosmology, Springer, 2010.

[7] D. Ghosh, A. Deb, S. Pal et al., "Evidence of fractal behavior of pions and protons in high energy interactions - an experimental investigation," Fractals, vol. 13, no. 4, pp. 325-339, 2005.

[8] A. N. Kolmogorov, "Local structure of turbulence in an incompressible viscous fluid at very high Reynolds numbers," Soviet Physics Uspekhi, vol. 10, no. 6, pp. 734-736, 1968.

[9] J. Qian, "Generalization of the Kolmogorov -5/3 law of turbulence," Physical Review E, vol. 50, no. 1, pp. 611-613, 1994.

[10] C. Brun, D. Juvé, M. Manhart, and C. D. Munz, Numerical Simulation of Turbulent Flows and Noise Generation, Springer, 2009.

[11] W. Hillebrandt and F. Kupka, Interdisciplinary Aspects of Turbulence, Springer, Berlin, Germany, 2009.

[12] R. Gomes-Fernandes, B. Ganapathisubramani, and J. C. Vassilicos, "Particle image velocimetry study of fractal-generated turbulence," Journal of Fluid Mechanics, vol. 711, pp. 306-3336, 2012.

[13] J. L. Lumley and A. M. Yaglom, "A Century of Turbulence," Flow, Turbulence and Combustion, vol. 66, no. 3, pp. 241-286, 2001.

[14] Z. Warhaft, "Turbulence in nature and in the laboratory," Proceedings of the National Academy of Sciences of the United States of America, vol. 99, no. 1, pp. 2481-2486, 2002.

[15] P. Geipel, K. H. H. Goh, and R. P. Lindstedt, "Fractal-generated turbulence in opposed jet flows," Flow, Turbulence and Combustion, vol. 85, no. 3-4, pp. 397-419, 2010.

[16] B. B. Mandelbrot, Gaussian Self-Affinity and Fractals, Springer, New York, NY, USA, 2002.

[17] B. B. Mandelbrot, The Fractal Geometry of Nature, W. H. Freeman, New York, NY, USA, 1982.

[18] C. Cattani, G. Pierro, and G. Altieri, "Entropy and multifractality for the myeloma multiple TET 2 gene," Mathematical Problems in Engineering, vol. 2012, Article ID 193761, 14 pages, 2012.

[19] C. Cattani, "Fractional calculus and Shannon wavelet," Mathematical Problems in Engineering, Article ID 502812, 26 pages, 2012.

[20] E. C. Ackermann, “The Golden Section,” The American Mathematical Monthly, vol. 2, no. 9-10, pp. 260-264, 1895.

[21] B. Kaygn, B. Balçin, C. Yildiz, and S. Arslan, "The effect of teaching the subject of Fibonacci numbers and golden ratio through the history of mathematics," Procedia-Social and Behavioral Sciences, vol. 15, pp. 961-965, 2011.

[22] S. Davies and P. Hall, "Fractal analysis of surface roughness by using spatial data," Journal of the Royal Statistical Society B, vol. 61, no. 1, pp. 3-37, 1999.
[23] T. Gneiting and M. Schlather, "Stochastic models that separate fractal dimension and the Hurst effect," SIAM Review, vol. 46, no. 2, pp. 269-282, 2004.

[24] R. P. Kanwal, Generalized Functions: Theory and Applications, Birkhäuser, 3rd edition, 2004.

[25] I. M. Gelfand and K. Vilenkin, Generalized Functions, vol. 1, Academic Press, New York, NY, USA, 1964.

[26] M. Li and S. C. Lim, "A rigorous derivation of power spectrum of fractional Gaussian noise," Fluctuation and Noise Letters, vol. 6, no. 4, pp. C33-C36, 2006.

[27] G. Chan, P. Hall, and D. S. Poskitt, "Periodogram-based estimators of fractal properties," The Annals of Statistics, vol. 23, no. 5, pp. 1684-1711, 1995.

[28] A. S. Monin and A. M. Yaglom, Statistical Fluid Mechanics: Mechanics of Turbulence, vol. 1, The MIT Press, Cambridge , Mass, USA, 1971.

[29] A. S. Monin and A. M. Yaglom, Statistical Fluid Mechanics: Mechanics of Turbulence, vol. 2, The MIT Press, Cambridge , Mass, USA, 1971.

[30] C. Tropea, A. L. Yarin, and F. John Foss, Springer Handbook of Experimental Fluid Mechanics, Springer, 2007. 

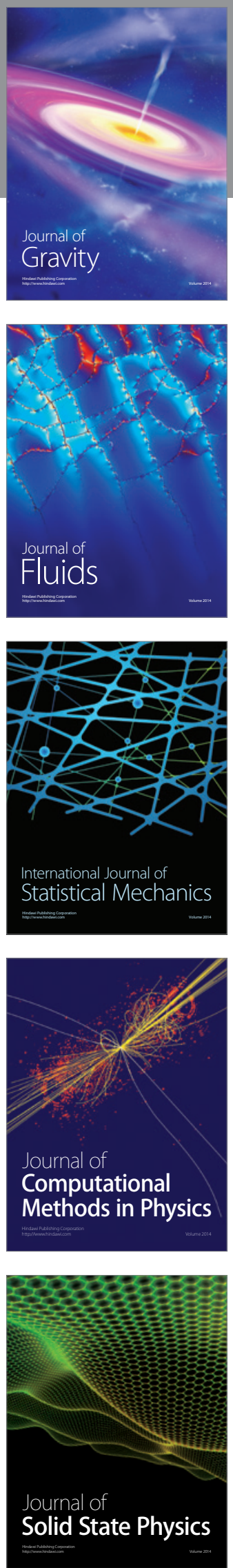

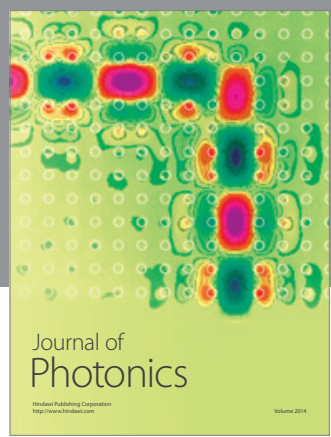

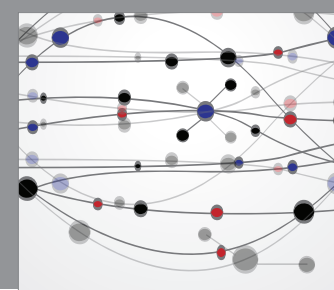

The Scientific World Journal

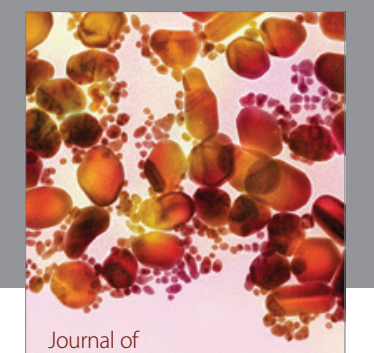

Soft Matter
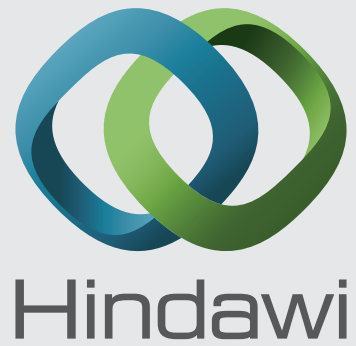

Submit your manuscripts at

http://www.hindawi.com
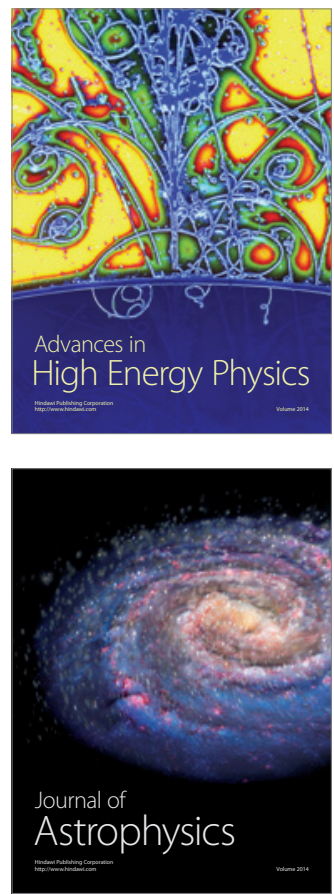
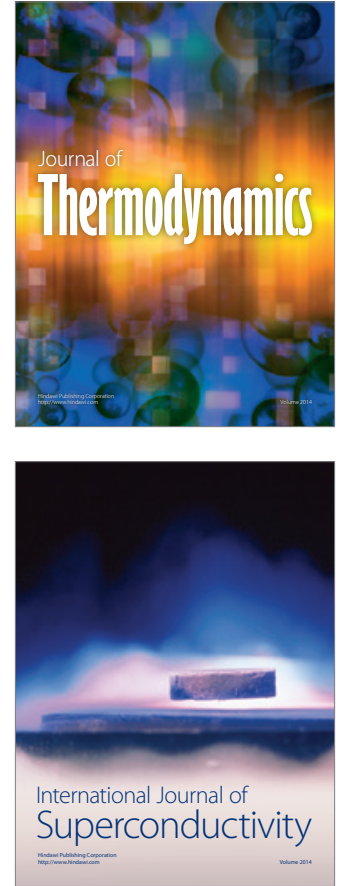
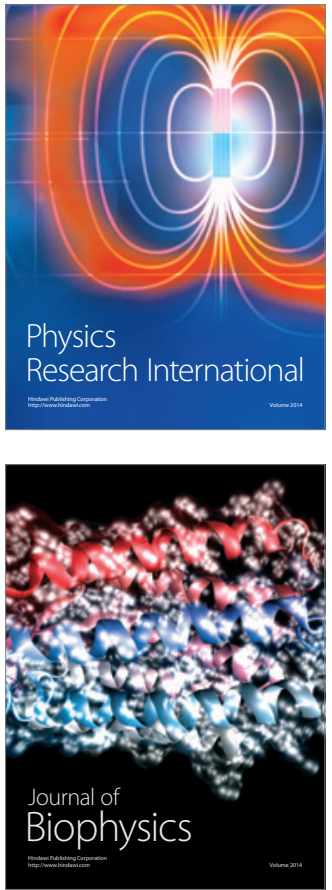
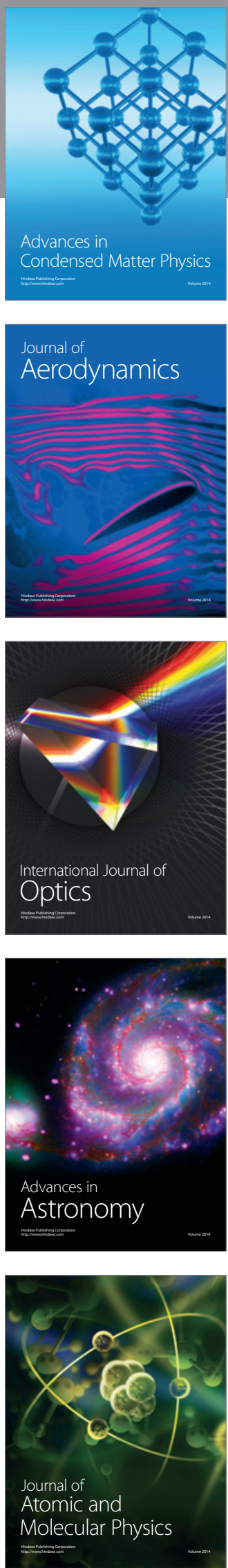